\title{
The treatment of mental contamination: a case series
}

Article

Accepted Version

Coughtrey, A. E., Shafran, R., Lee, M. and Rachman, S. (2013) The treatment of mental contamination: a case series. Cognitive and Behavioural Practice, 20 (2). pp. 221-231. ISSN 1077-7229 doi: https://doi.org/10.1016/j.cbpra.2012.07.002 Available at https://centaur.reading.ac.uk/31316/

It is advisable to refer to the publisher's version if you intend to cite from the work. See Guidance on citing.

To link to this article DOI: http://dx.doi.org/10.1016/j.cbpra.2012.07.002

Publisher: Elsevier

All outputs in CentAUR are protected by Intellectual Property Rights law, including copyright law. Copyright and IPR is retained by the creators or other copyright holders. Terms and conditions for use of this material are defined in the End User Agreement.

\section{www.reading.ac.uk/centaur}

\section{CentAUR}

Central Archive at the University of Reading

Reading's research outputs online 


\begin{abstract}
The recommended treatment for Obsessive Compulsive Disorder (OCD) is Cognitive Behaviour Therapy (CBT) incorporating Exposure and Response Prevention (ERP) which is effective for approximately $50 \%$ of patients. However, there has been little advance in treatment outcomes since the introduction of ERP in 1979. It has been suggested that some progress can be made in treating contamination obsessions and washing compulsions by addressing feelings of dirtiness and contamination that arise without physical contact with a tangible contaminant. To date, the treatment of these 'mental contamination' fears in OCD have not been systematically explored. This paper reports a case series of 12 participants with OCD who received 10-20 sessions of a CBT based treatment for mental contamination, and describes and analyses the treatment. At the end of treatment, 7 participants no longer met the diagnostic criteria for OCD and mental contamination and these gains were maintained at 6 month follow-up. The clinical implications of these findings are discussed.
\end{abstract}

KEYWORDS: Contamination, mental contamination, Cognitive Behavioural Therapy (CBT), Exposure and Response Prevention (ERP) 


\section{Introduction}

OCD is a disabling condition (Bobes, Gonzalez, Bascaran, Arango, Saiz \& Bousono, 2001) and is considered to be the most serious of the anxiety disorders (Kessler, Chui, Demler \& Walters, 2005). Psychological treatment (predominantly CBT incorporating ERP) is moderately effective (NICE, 2005), but the percentage of positive outcomes has not increased over the past 40 years (Rachman, 2006) and there is a pressing need for improvements in treatment efficacy (Sookman \& Steketee, 2007; Wilhelm, 2000). The repeated, controlled, planned and deliberate exposure to frightening stimuli, while inhibiting any neutralising response (ERP) is typically the primary component of treatment. Many patients refuse treatment or drop out (e.g. 43\%, Foa et al., 2005), or do not improve or relapse by follow-up (Jenike, Baer \& Minichiello, 1998; Clark, 2004; Cottraux, Bouvard \& Milliery, 2005; Stanley \& Turner, 1995).It is a demanding treatment and can be difficult to tolerate. The available figures suggest that only $25-50 \%$ of patients who complete treatment make a full recovery (Abramowitz, 2006; Eddy et al., 2004; Fisher \& Wells, 2005; Foa et al., 2005; Whittal, Robichaud, Thordarson \& McLean, 2008). Even after treatment many patients report that OCD continues to have a severe impact on their social functioning, familial relationships and their ability to work and study (Niederauer, Braga, de Souza, Meyer \&Cordoli, 2007). According to Niederauer and colleagues (2007), these impairments are comparable to those experienced by patients with schizophrenia. Therefore, evidently there is a need to develop more effective and tolerable treatments for patients with OCD.

It has been proposed that the second most common form of OCD, cleaning compulsions, can be treated more effectively by recognising the occurrence of 'mental contamination' and treating it with CBT (Rachman, 2006). In contrast to the familiar phenomenon of contact contamination, mental contamination (also known as emotional contamination) consists of feelings of contamination and dirtiness that arise without physical 
contact with a tangible stimulus. The source of contamination is human, rather than inanimate, and the feelings of dirtiness and pollution are predominantly internal, and consequently less amenable to exposure treatment in which the person is repeatedly exposed to tangible contaminating stimuli, such as dirt, disease and chemicals, for prolonged periods.

It is proposed that some progress can be made by recognising and treating mental contamination. These fears have a human source in which psychological or physical violation has taken place. They can be evoked without physical contact with a contaminant, are idiosyncratic and evocable by memories, images, thoughts, remarks. The feelings of contamination are internal as well as external, and unresponsive to washing. People with mental contamination may experience morphing or 'transformation obsessions' (Volz \& Heyman, 2007) where they fear they may take on the undesirable characteristics of an unsavoury person(s) and in extreme cases, turn into them. The type of person classed as undesirable is both personally and culturally defined but may include personal enemies or people who are considered weird, dirty or of low status (Rachman, 2006).

Mental contamination is thought to be associated with Thought-Action Fusion (TAF), 'the belief that thinking about an unacceptable or disturbing event makes it more likely to happen, and the belief that having an unacceptable thought is the moral equivalent of carrying out the unacceptable or disturbing action' (Shafran, Thordarson \& Rachman, 1996; page 379). TAF is believed to contribute to the maintenance of mental contamination by raising estimations of the probability and severity of potential harm and increasing feelings of harm, guilt and responsibility, as well as contributing to the inference that danger and contamination is present (Rachman, 2006; Coughtrey, Shafran, Knibbs \& Rachman, submitted). In some cases, the feelings of guilt, anxiety and low mood that result from TAF and external triggers can be misinterpreted as feelings of dirtiness or pollution. Such mislabelling of mood states 
may form an important component of treatment, particularly in those with comorbid disorders.

Mental contamination is thought to be common; in a study of 177 people with OCD symptoms it emerged that $46 \%$ of them experienced mental contamination (Coughtrey et al., submitted).Mental contamination was highly correlated with severity of the OCD. In an expanded version of this study, nearly half of the 54 participants who had moderate-severe OCD and were receiving treatment, reported mental contamination. Until recently the presence of mental contamination in patients with OCD was neither recognised nor treated.

We have previously suggested that cognitive approaches to treatment are more fitting for dealing with mental contamination than behavioural ERP methods (Rachman, 2006). We have proposed that the focus should be on cognitive processes, augmented by behavioural experiments to test these key cognitions, as compared to the traditional exposure work used in the treatment of familiar contact contamination. A variety of cognitive interventions are used in modifying the maladaptive cognitions, such as changing the meaning and interpretation of the sources of contamination, modifying self-generated contamination by helping the patient recognise the effects of maladaptive misinterpretations of the significance of the feelings of contamination, and by separating feelings of anger, aversion, and disgust from mental contamination. A preliminary case study has shown that these are effective techniques in reducing crippling levels of mental contamination fear (Warnock-Parkes, Salkovskis \& Rachman, 2011). In cases where there is an overlap between mental and contact contamination, treatment techniques which address both forms of contamination fears are required.

The aim of this investigation was to study the effects of a specific, theory-driven cognitive-behavioural intervention for mental contamination. CBT has proven effective in 
treating other manifestation of OCD, notably obsessions (e.g. Freeston et al., 1997; Salkovskis, 2007; Whittal, Woody, McLean, Rachman \& Robichaud, 2010), and it was hypothesised that a similar approach can produce comparable reductions in mental contamination. It was predicted that following treatment, the participants would report clinically significant decreases in mental contamination, OCD symptoms, TAF and anxiety.

\section{Method}

\section{Participants}

Patients with OCD of clinical severity and whose primary problem was mental contamination were selected for participation in the study. The inclusion/exclusion criteria were: aged between 18-65 years, suitable for treatment as an outpatient, medically stable, no significant suicide risk and no psychotic illness. Participants with co-morbid diagnoses were not excluded.

Participants were referred from local NHS outpatient services, local counselling teams and support groups. In total, 29 potential participants were referred for treatment. Of these people, eight were excluded because they did not attend the assessment session or did not wish to receive treatment as part of a research trial, seven did not have mental contamination concerns and two were excluded because they required inpatient care. There were no significant differences between participants who were included and excluded from the study in terms of gender, $\chi^{2}(1, N=29)=3.62, p=.07$, age, age of onset, duration of illness or OCD symptom severity, all $p$ 's $>.05$. Participants who were included had significantly higher scores on a measure of mental contamination than participants who were excluded, $t$ $(27)=2.31, p=.03, d=.95 ;$ Mean $=57.92, S D=15.49$ vs. Mean $=34.33, S D=31.72$ respectively. 
The 12 participants who began treatment consisted of five males and seven females, with a mean age of 28.83 years $(S D=8.54)$. The average age of onset of OCD symptoms was 18.73 years $(S D=5.55)$ and participants had experienced OCD symptoms for an average of 11.55 years $(S D=9.06)$. The average OCI-R score was $42.67(S D=10.98)$ indicating that participants had OCD of moderate-severe severity (Foa et al., 2002). All participants had failed to respond to some form of psychological intervention for their OCD symptoms in the past (nine had received a course of CBT involving ERP), and four participants were receiving pharmacological treatment for their OCD at the start of treatment. No participant initiated or continued in another form of psychological or pharmacological therapy during treatment or follow-up.

\section{Procedure}

All participants were offered an appointment to discuss the treatment trial within three weeks of referral and were given the opportunity to discuss the study with friends, family and health care professionals before providing written consent to participate. Participants received between 10-20 fifty minute treatment sessions delivered jointly by RS and AC. Participants attended a ninety minute research assessment conducted by an independent clinician prior to the intervention and at the end of treatment. Participants were contacted approximately 3 months and 6 months after the last treatment session to obtain follow-up data; eight participants completed a 3 month follow-up and seven completed a 6 month follow-up.

\section{Measures}

\section{Anxiety Disorders Inventory Schedule for DSM-IV (ADIS-IV; Brown, DiNardo}

\& Barlow, 1994). This is a widely used semi-structured diagnostic interview which assesses current episodes of anxiety disorders based on DSM-IV criteria (American Psychiatric 
Association, 1994). Participants completed the ADIS-IV with an independent clinician at pre-treatment, post-treatment and follow-up.

Yale-Brown Obsessive-Compulsive Scale - Interview Version (Y-BOCS;

Goodman, Price, Rasmussen, Mazure, Fleischmann, Hill, Heniger \& Charney, 1989).

The Y-BOCS is a widely used, clinician administered interview designed to assess the severity of both obsessive and compulsive symptoms. There are two five item subscales: one for obsessions and one for compulsions; the total score (ranging from 0-40) is based on the total of these two subscales. The Y-BOCS has good inter-rater and test-retest reliability over one week (Goodman et al., 1989; Kim, Dysken \& Kuskowski, 1990).

\section{Vancouver Obsessional Compulsive Inventory-Mental Contamination Scale}

(VOCI-MC; Rachman, 2006). This 27 item scale assesses aspects of mental contamination. Participants rate each item e.g. e.g. "I often feel dirty under my skin" on a five point scale from $0=$ not at all, to $4=$ very much. The VOCI-MC has high internal consistency (Cronbach's $\alpha=$.94; Rachman, 2006; Radomsky\& Elliott, 2009).

\section{Obsessional Compulsive Inventory Short Version (OCI-R; Foa, Huppert,} Leiberg, Langner, Kichic et al., 2002). This is a shortened version of the original OCI, designed to assess OCD symptomatology and severity. It contains 18 items, e.g. "I wash my hands often and longer than necessary" that participants rate on a five point scale from $0=$ not at all to $4=$ extremely. The OCI-R is a psychometrically sound measure with reported internal consistency, test-retest reliability and discriminant validity (Foa et al., 2002).

\section{Thought Action Fusion Scale (TAF; Shafran, Thordarson \& Rachman, 1996).} This 19 item instrument is used to assess TAF. Participants rate statements e.g. "If I wish harm on someone, it is almost as bad as doing harm" on a five point scale from $0=$ strongly disagree, to 4 = strongly agree. The TAF scale contains items pertaining to moral TAF, and 
items related to harm to self, and harm to others and has good internal consistency ( $\alpha=.92$; Shafran et al., 1996).

Beck Anxiety Inventory (BAI; Beck \& Steer, 1990). This 21 item instrument is used as a measure of clinical symptoms of anxiety and is reported to have good internal consistency $(r=.92$; Beck \& Steer, 1990).

Beck Depression Inventory (BDI-II; Beck, Steer \& Brown, 1996). This 21 item self report instrument is used to measure depressive symptoms over the past two weeks. The BDI-II has high internal consistency, as indicated by reported alpha coefficients of .90 (Beck, Steer, Ball \& Ranieri, 1996) and one week test-retest reliability $(r=.93$; Beck, Steer $\&$ Brown, 1996).

\section{Treatment Protocol}

The treatment protocol used in this intervention was based on the cognitivebehavioural theory of mental contamination (Rachman, 2006). Participants received between 10-20 fifty minute sessions of individual CBT delivered jointly by RS and AC. Based on the treatment guidelines for moderate to severe depression and on previous research into interventions for bulimia and perfectionism (Agras, Walsh, Fairburn, Wilson \& Kraemer, 2000; NICE, 2009; Riley, Lee, Cooper, Fairburn \& Shafran, 2007), the first six sessions were delivered twice weekly, before switching to weekly sessions and finally to once fortnightly for the last 2-3 treatment sessions.

The process of treatment is the same as CBT for contact contamination, but the content is different and tailored specifically for mental contamination. No ERP was used in this treatment. In addition to generic therapeutic and CBT skills such as engagement, setting goals, instilling hope and reflecting and summarising, treatment was tailored specifically for mental contamination as outlined below. 
1) Assessment. Throughout the assessment, the therapist worked with the client to make sense of the problem. This involved the following:

- Understanding the current problem and its impact in detail. Asking for a specific and recent example of mental contamination was used to elicit thoughts, feelings and counter-productive behaviours, e.g. questions such as 'what do you do to cope with your fears?'

- Gathering information about source(s) of contamination in particular human sources and hypervigilance to these sources. This included asking questions about vulnerability to morphing (taking on the undesirable characteristics of another person), e.g. 'are you worried you might become like them?' and 'how would that happen?' as well as ascertaining whether the person believed that they were able to take on the positive characteristics of a desirable person e.g. 'can you ever pick up positive characteristics?'

- Taking a detailed history of the development of mental contamination including questions about when the problem started, speed of onset, how the client makes sense of the problem and personal vulnerability. This included questions such as 'how do you make sense of the problem?', 'if that happened to someone else, do you think they would become contaminated?', 'what was happening in your life when the problem first started?' and 'what would be the worst outcome?'

- A focus on previous or current physical and psychological violations and betrayals (Rachman, 2010). This typically began by asking clients 'can you tell me about anyone who has been particularly helpful to you? What were their characteristics?' before asking them questions such as 'can you tell me about anyone who has been particularly unhelpful to you? You don't have to identify them if you don't wish to. What were their characteristics?' 
- Obtaining information about the nature of the spread of mental contamination e.g. 'do new items/persons/places ever become contaminated? How do they become contaminated?'

- A focus on mental imagery e.g. asking clients 'are there any pictures that cause you to feel contaminated?' This also included questions about protective images e.g. 'are there any pictures in your mind that you use to protect yourself?' Some patients reported protective imagery shields, which were explored further including questions about the effort it took to create the protective shield and its effectiveness.

2) Formulation. An individualised formulation of the maintenance of the problem was devised, based on the theory of mental contamination (Rachman, 2006) and cognitivebehavioural model of OCD (Whittal, 2010). The formulation focused on triggers of mental contamination (including imagery), beliefs relating to contamination and maintaining behaviours (e.g. compulsive washing and avoidance). A historical formulation was constructed to help the client make sense of the problem (Beck, 1976).

3) Psychoeducation. A semi-didactic component to provide psychoeducation and corrective information about mental contamination was included. Clients were asked about what they knew about OCD and contamination fears. The different types of contamination were described to clients, including using descriptions used by previous clients e.g. 'there is another kind of contamination where people experience very similar feelings (to contact contamination) like feeling dirty or polluted without touching anything. This kind of contamination is not localised to your hands but is diffuse within the body. Some people describe it like having "mind germs" because certain thoughts and images can convey the feeling of contamination.' 
The human source of contamination was also explained to clients e.g. 'mental contamination invariably starts from a person(s). People who are afflicted with mental contamination have sometimes experienced physical or emotional violation, for example a betrayal of a good friend, domination by a parent, or an assault and this can sensitise them to fears of contamination. The fears can spread and generalise by becoming associated with particular categories of people.' The role of concealment in maintaining appraisals was also highlighted to clients.

A further area of discussion was mislabelling mood states, separating feelings of anger, aversion and disgust from feelings of mental contamination and explaining the exconsequentia reasoning bias to clients e.g. 'some people reason that if they feel guilty, they must have done something wrong and therefore they think "I am contaminated." Sometimes when people think they are feeling dirty or polluted they are actually also feeling sad or angry or disgusted but have mislabelled the emotion and that's why sometimes washing doesn't help you feel better.'

4) Monitoring. Clients were asked to monitor and record contaminating triggers (in particular human sources and their own thoughts and images), the intensity of mental contamination, location of pollution within themselves, and subsequent behaviour e.g. forming a protective image, avoidance, washing, drinking water. The particular behaviour that was monitored was derived from the individualised formulation. Personalised sessionby-session monitoring of symptoms was utilised throughout treatment e.g. recording the frequency and intensity of contaminating and protective images.

5) Surveys. Surveys were used to collect personally relevant information. In some cases this was used to normalise the fears, as with traditional CBT, e.g. by asking people how they would feel wearing a dead man's clothing. In other cases the survey was related to moral 
standards, reaction to particular categories of people or ways of coping with emotions such as guilt and depression.

6) Behavioural experiments. Behavioural experiments formed a fundamental component of treatment. They were used to test beliefs about contamination and reduce maintaining behaviours, e.g. avoidance and washing behaviours, in order to provide the patient with direct personal evidence about their maladaptive cognitions and behaviours. Although the behavioural experiments may have involved exposure, the exposure was not prolonged, repeated or graded. The experiments were designed to change beliefs and no ERP was used in this treatment.

Behavioural experiments were particularly useful for exploring TAF and morality which are common problems in mental contamination. For example these included a modification of Rachman and colleagues (1996) experiment in which clients are asked to write down a sentence wishing harm on a loved one to explore the influence of TAF on mental contamination. In addition, following from a survey about appropriate moral standards, the client would do an experiment where they would act 'as if' they had the same moral standards as others on one day and then on the next day they would revert to their own (usually higher) moral standards. Feelings of contamination on the contrasting days would be assessed and conclusions drawn about the impact of high moral standards on their contamination fears.

7) Addressing the meaning of contamination. Cognitive-behavioural techniques were used to change the meaning of the source of contamination, modify self-generated contamination by reinterpreting the significance of the feelings of contamination, address the meaning of dirtiness and link this to issues of self-esteem and self-identity. Typical methods would include helping the client see it from another perspective through discussion and cognitive 
restructuring. This also included experiments where the client and therapist compare two theories for the reason for feeling contaminated e.g. deliberately trying to spread contamination to test whether the person was truly contaminated or whether it was a problem of their thinking. This has been termed 'Theory A' and 'Theory B' (Salkovskis \& Kirk, 1997). Issues of self-identity were particularly relevant when tackling beliefs about morphing. Clients completed behavioural experiments to reinforce their self-identity and increase self-esteem and thus reduce their vulnerability to morphing fears e.g. making a list of personal characteristics that were stable and unchanging.

8) Imagery. A significant amount of treatment was devoted to understanding the role of images in triggering and maintaining mental contamination. Initially it was assessed in session using the following test. Clients were asked to rate their current feelings of contamination from 0-100 and then asked to close their eyes and form a picture of their best friend and think about him/her as clearly as they could. They were asked to raise their hand when they had formed a vivid image in their mind and to keep thinking about this person. Clients then re-rated their contamination levels. Clients were then asked to close their eyes again and to 'imagine you are walking down the street, you turn the corner and you see (source of contamination) or have a contaminating thought.' Clients were asked to re-rate their feelings of contamination and describe how they were feeling e.g. "where in your body do you feel the contamination? When you feel like this what do you have the urge to do?'

Clients were encouraged to drop protective images in a behavioural experiment that assessed whether the image actually prolongs feelings of contamination in the long term. They were encouraged to distance themselves from any images that are causing feelings of contamination and rescript them (e.g. see Wild \& Clark, 2011). 
9) Relapse prevention. An individualised relapse prevention plan, which included describing the factors that had maintained their contamination fear, the techniques the client had found useful in treatment, identifying potential triggers for setbacks, and considering ways of resolving future difficulties was drawn up with the client.

Family, friends and carers were involved in formulation and treatment when appropriate. Clients completed a number of tasks between sessions in order to gather information about the nature of their mental contamination fears and to test competing hypotheses about their beliefs.

\section{Case Descriptions}

The treatment of mental contamination is illustrated with two cases, one with a positive outcome and one with limited success. To protect participants' anonymity, their initials and any key identifying information have been changed. For outcome data pertaining to these cases, please see Table 1.

Case 12. Assessment. C was a 34 year old Caucasian woman with a 27 year history of contamination concerns and compulsive washing. Her OCD symptoms centred on preventing future harm to herself and her loved ones, which led her to repeat actions when she had a bad thought and to replace bad thoughts with positive ones in order to prevent the bad thought travelling with her and contaminating other people and items. She described feeling as if she had badness all over her skin and feared she could transmit the badness to others via her thoughts, although she recognised that this thinking was magical. $\mathrm{C}$ also reported having a number of mental shields which she used to protect herself, her possessions and her loved ones from badness. In addition, $\mathrm{C}$ reported that she was vulnerable to morph into people she deemed undesirable, or that her loved ones would dislike or pity e.g. obese people or alcoholics, and described noticing magical omens relating to her fear of morphing. 
She washed herself and her clothes if she came into contact with these people or omens. She also reported an inability to discuss the future, as she felt that she might contaminate it and by discussing it, would be inviting something bad to happen. This had recently led to the failure of her long-term relationship. In the past $\mathrm{C}$ had received 6 sessions of individual $\mathrm{CBT}$, with minimal benefit.

Conceptualisation. C explained how as a child she felt overpowered by her mother who she described as 'overbearing.' She talked about how her childhood experiences had left her feeling vulnerable to potential negative life events and unable to cope with adversity. She discussed how this left her feeling like she must always prevent future harm to herself and her loved ones. In addition, C recalled a memory of playing 'tag' in the playground at school when she felt as though she had caught some 'bugs'. She explained that since this time she had obliged to prevent the future transmission of contamination and badness.

There were multiple triggers which would result in $\mathrm{C}$ experiencing intrusive thoughts of future harm and contamination. C would then interpret these thoughts as 'I'm/it's contaminated' and believed that she must prevent the bad future consequences by stopping the contamination spreading. This led to extreme feelings of dirtiness and pollution and washing behaviours. In addition, $\mathrm{C}$ would construct a mental shield to protect herself from morphing. This was conceptualised as maintaining her beliefs that she was vulnerable to change and responsible from preventing future harm.

Treatment. C completed 16 sessions of treatment over which she made excellent progress. Initially, the main focus of treatment was addressing C's beliefs about contaminating the future, by using a series of surveys and behavioural experiments e.g. asking $\mathrm{C}$ to deliberately try and contaminate an event that was happening the next day. This enabled $\mathrm{C}$ to establish that the problem was not that she could contaminate the future, but that 
it was a problem of her thinking. The focus of the remaining sessions was on C's mental shield that she constructed to cope with her feelings of mental contamination. Monitoring her shield and conducting an experiment within a treatment session where she compared a contaminating encounter with and without her shield, enabled her to realise that rather than protecting her from feeling contaminated, constructing the shield maintained her focus on her feelings of contamination and thus prolonged her anxiety and feelings of pollution. As a result of this, $\mathrm{C}$ began to conduct behavioural experiments in which she would come into contact with places, people and objects that she had previously avoided, both with and without using her shield. Over time, she was able to minimise the use the mental image of the shield, and this enabled her significantly to reduce the avoidance. The final section of treatment tackled C's beliefs about social judgement and her feeling of being vulnerable to morphing because of her unstable sense of self. General cognitive and behavioural techniques to improve self-esteem were used, and C started to generate a list of things about her that were stable and not subject to change. $\mathrm{C}$ was introduced to formal problem solving techniques to enable her to start to feel that she had the capacity to cope in the event of general negative life events occurring in the future.

Outcome. At the end of treatment $\mathrm{C}$ no longer met the diagnostic criteria for any mental health problem. She no longer avoided large areas of her local town, and was able to talk freely about the future and have physical contact with her loved ones. These gains were maintained at 3 and 6 month follow-up.

Case 8. Assessment. T was a 43 year old Asian female with an 18 year history of OCD symptoms related to a fear of contamination associated with faeces. T demonstrated mental contamination in that even imagining faeces would cause her to feel dirty and wash compulsively. At the beginning of treatment, T reported excessive washing behaviour including using two bottles of shower gel per day, and double washing all her clothes using 
five washing tablets per wash. Thad not slept in her own bed for over 10 years for fear of spreading contamination. Related to her contamination fears, T presented with low mood, low self-esteem and frequent panic attacks.

T had received individual CBT for her contamination fears on two past occasions, once for 14 sessions and the other 16 sessions. Treatment on one of these occasions was with an accredited BABCP therapist.

Conceptualisation. For T, the term dirty was intertwined with pathetic and useless, resulting from an unhappy marriage during which she suffered physical violence and humiliation. T would often experience an intrusive mental image of a time when she had previously been abused and this left her feeling dirty and contaminated as she was concerned she may have soiled herself. In addition bodily sensations and anything she saw that resembled faeces would trigger intrusive thoughts that she had soiled herself, which would leave her feeling dirty, pathetic and useless. As a result, T engaged in compulsive washing and avoidance.

Treatment. T completed 14 sessions of treatment during which she made some improvement with regard to her contamination fear. Discussion of betrayal and humiliation was particularly important with $\mathrm{T}$, in order to establish that the term dirty was intertwined with pathetic and useless. Treatment involved cognitive work along with behavioural experiments to demonstrate that feelings of humiliation and betrayal could make her feel dirty e.g. by asking her in sessions to imagine times when she had been betrayed and monitoring her subsequent feelings of pollution. Similarly, cognitive techniques were used to allow $\mathrm{T}$ to reinterpret the significance of the feelings of contamination, to address the meaning of dirtiness and to separate anger, aversion and feeling pathetic and useless from feelings of contamination. This involved focusing on all or nothing thinking with regard to 
feeling pathetic and useless and establishing that there were other ways to feel better rather than needing to feel clean. $\mathrm{T}$ also completed a number of tasks to improve her self-esteem e.g. noticing and recording things that were good about herself.

Outcome. By the end of treatment, $\mathrm{T}$ had made some significant improvements, for example, she was able to sleep in her own bed. However, she still met the diagnostic criteria for OCD and continued to experience crippling contamination fears. She was referred for psychotherapy and work on self-esteem problems.

\section{Case Series Results}

At post-treatment assessment, seven out of twelve participants no longer met the diagnostic criteria for OCD as defined by the ADIS-IV. These gains were maintained at follow-up. The remaining five participants still met the diagnostic criteria for OCD; two of these participants no longer experienced mental contamination.

Table 1 shows the scores for each participant at pre-treatment, post-treatment, 3 month follow-up and 6 month follow-up. Follow-up data are missing for participants 2, 4, 5, 8 and 10. Participants who demonstrated clinically significant change as defined by Jacobson and Truax (1991) definition c - 'the level of functioning subsequent to therapy places that client closer to the mean of the functional population than it does to the mean of the dysfunctional population' - are highlighted.

\section{--INSERT TABLE 1 HERE---}

Not surprisingly, the seven participants who no longer met the diagnostic criteria for OCD showed clinically significant improvements on the Y-BOCS and the OCI-R. These gains were maintained over 3 and 6 month follow-up. Nine participants demonstrated clinically significant reductions in mental contamination and TAF, gains which again were 
maintained over the follow-up period (data missing for two participants). Seven participants showed a significant reduction in symptoms of anxiety, and six a reduction in depressive symptoms.

Table 2 shows the mean scores pre and post-treatment and for follow-up. Paired samples $t$-tests revealed significant group reductions for all the measures pre to posttreatment: Y-BOCS, $t(11)=4.17, p=.002, d=1.55 ;$ OCI-R, $t(11)=5.42, p<.001, d=1.84$ VOCI-MC, $t(11)=5.94, p<.001, d=1.42 ;$ TAF, $t(11)=5.75, p<.001, d=2.24 ;$ BDI-II, $t$ $(11)=3.06, p=.01, d=.79$; and BAI, $t(11)=4.62, p=.001, d=.60$. There were no significant differences for any measure post-treatment compared to follow-up at 3 months or 6 months. All mean scores post-treatment were in the normal range, apart from the BDI-II and BAI (mild).

\section{Discussion}

The findings from this case series indicate that mental contamination is responsive to a cognitive-behavioural treatment. Seven of the 11 participants demonstrated a complete recovery from their OCD, and these gains were maintained over three and six month followup. Furthermore, nine participants demonstrated clinically significant reductions in mental contamination and TAF which also were maintained over the follow-up period. In addition, seven participants showed a significant reduction in symptoms of anxiety and six a reduction in depressive symptoms. As a group, there were significant reductions on all the individual measures of psychopathology with large effect sizes similar to those reported in trials of CBT for other forms of psychopathology (e.g. Chambless \& Gillis, 1993). These findings provide preliminary evidence that a cognitive-behavioural intervention designed specifically to target mental contamination is successful in reducing contamination fear, OCD symptoms, TAF, and associated anxiety and depression. The success rates reported here are promising 
compared to the finding that only $25 \%$ of patients with OCD demonstrate a complete recovery (Eddy et al., 2004), and are similar to the improved treatment recovery rates reported for pure obsessions (Freeston et al., 1997; Salkovskis, 2007; Whittal et al., 2010).

Those participants who were able successfully to carry out homework tasks, and build on the behavioural experiments and adapt them where necessary, made the greatest gains in treatment. Two participants completed treatment but did not show any clinically significant improvement in mental contamination or OCD symptoms. The reasons for lack of progress in these two cases included severe depression and suicidality and low self esteem which made it difficult for these participants to apply what they learned in-session to their home environments. It is noteworthy that these participants had experienced symptoms of mental contamination for a considerable length of time, and had both received CBT for their OCD on more than one occasion in the past. In addition, they had the highest scores on the VOCI-MC at the start of treatment, and one presented with co-morbid panic disorder. These findings suggest that people with long-standing complex mental contamination may benefit from a lengthier or different approach to treatment; alternatively it may be more appropriate to address comorbid issues prior to mental contamination.

\section{Implications for the Construct of Mental Contamination}

The findings of this case series suggest that the construct of mental contamination has clinical utility and that a brief CBT intervention can be effective in treating this client group, many of whom had failed to respond to previous psychological treatment. These results are promising but limited in size. The treatment was not specifically designed to target symptoms of depression or anxiety, but there were significant reductions in scores on the BDI-II and BAI were reported. Similarly, the reduction in TAF (which was a target of treatment) provides some supporting evidence for the role of the TAF bias in the maintenance of mental 
contamination fear (Rachman, 2006).Finally it is noteworthy that issues of morality were common across all cases in this treatment trial. Mental contamination is postulated to be closely associated with feelings and concerns about immorality, and future research would benefit from examining this aspect of contamination more closely.

\section{Implications for Clinical Practice}

The findings of this study have clear implications for clinical practice. First, the findings indicate that mental contamination can be responsive to CBT. A full and detailed assessment is important to identify all sources of contamination, both mental and contact, and to establish the degree of overlap. It may be helpful to use measures of mental contamination and TAF throughout treatment in order to track and monitor the sources and forms of mental contamination. It is advisable to ask questions about betrayal and morality, and to consider that feelings of contamination can be triggered in perpetrators as well as victims. For example, it is important to ask patients whether they have ever been betrayed, or whether they have betrayed someone else, and what their emotional reaction was to that betrayal (Rachman, 2010).

Assessing and modifying intrusive mental imagery was a valuable technique in treatment. The assessment of the images can be revealing (Beck, 1976) and also throw light on the maintenance of the feelings of pollution, dirtiness, guilt. Images can trigger and maintain feelings of dirtiness, but the rescripting technique was a powerful tool for reducing the intrusive negative images and changing their meaning. Issues of morality were common in all participants in this study, and it may be important to address these in clinical practice. One particularly useful tool was asking participants consider adopting the moral codes of someone that they admired in a behavioural experiment. Addressing self-esteem and stability of self-concept was also important. Although self-esteem is regularly addressed in routine 
clinical practice, it was particularly important in our cases of mental contamination to consider the stability of the sense of self as this appeared to contribute to some participants feeling vulnerable to morphing. Finally, TAF fears was a common feature of all the cases reported in this study and were addressed using behavioural experiments that involved contamination-specific fears, for example deliberately trying to contaminate an object without touching it.

\section{Limitations and Future Research}

Further research is needed to refine and standardise the treatment protocol so that subsequent treatment interventions and research can a) identify the key components that are important in the intervention's success; b) develop effective training in order that other therapists can administer the treatment; and c) include measures of adherence to protocol and the influence on treatment outcome.

A limitation of this study is the case series design. The absence of a baseline or waitlist control means that the possibility that the reductions in symptoms are due to spontaneous remission or the natural passage of time cannot be ruled out. However, this explanation is not likely given that all participants had received some form of intervention prior to the start of treatment, and that they had experienced a fear of mental contamination for a considerable length of time. Additionally it is possible, although again unlikely given participants' past treatment histories, that the gains made in treatment were due to the therapeutic alliance with the therapist rather than the cognitive-behavioural intervention per se. Finally, it is important to note that the treatment reported here was conducted by an experienced clinician in a research setting, in contrast to participants' previous treatment in a clinical setting. Therefore these findings may not be generalisable to other people with mental contamination concerns in routine clinical settings and the effectiveness of the 
treatment may in part be attributable to the research setting. Further research is needed to explore these issues in more depth.

In this study treatment outcome was measured by clinically significant change on questionnaire and interview measures administered pre and post-treatment. Future research would benefit from including session-by-session measures to assess change and other measures of mental contamination e.g. behavioural tasks and measures of avoidance, neutralisation and compulsive washing in order to assess treatment outcome more fully. In addition the use of session-by-session measures would allow exploration of whether changes in mental contamination preceded broader symptom change. Furthermore, the sample reported here require longer follow-up in order to establish the gains seen in treatment. The current treatment recommendations for OCD stipulate that patients should be reviewed regularly for 12 months after discharge (NICE, 2005), and although this was offered to all participants, research assessments ceased after 6 months. Future research should therefore follow-up participants for at least one year post treatment.

The theory of mental contamination was developed in part to improve treatment outcome for people with OCD (Rachman, 2006). The preliminary findings reported here indicate that an intervention designed to specifically target mental contamination was successful for a number of people, all of whom had received psychological interventions for their OCD in the past. Although participants informally reported that they found treatment acceptable and beneficial, direct feedback about treatment acceptability was not formally obtained. Further research would benefit from exploring this and examining the components of treatment participants find most useful. Finally, full scale randomised control trials (RCTs) will be required to compare the CBT intervention for mental contamination with standard CBT for contamination fears as well as with a waitlist sample and an alternative therapy (such as cognitive therapy or interpersonal therapy). This would crucially contribute 
to demonstrating the effectiveness of the focused CBT reported here in modifying mental contamination.

\section{Conclusions}

A specific cognitive-behavioural based intervention for mental contamination was found to result in clinically significant change in contamination fear for 9 participants, and at post-treatment 7 participants no longer met the diagnostic criteria for OCD. These gains were maintained at 3 and 6 month follow-up. This is an important demonstration that mental contamination can be successfully treated using a modified form of CBT for OCD and has important implications for the understanding and treatment of contamination fear. Further research is needed to ensure that these gains are maintained over a longer follow-up period, and to compare the efficacy of the treatment with waitlist and standard CBT. 


\section{References}

Abramowitz, J.S. (2006). The psychological treatment of obsessive-compulsive disorder. Canadian Journal of Psychiatry, 51, 407-416.

Agras, W.S., Walsh, B.T., Fairburn, C.G., Wilson, G.T., \& Kraemer, H.C. (2000). A multicenter comparison of cognitive-behavioural therapy and interpersonal psychotherapy for bulimia nervosa. Archives of General Psychiatry, 57, 459-466.

American Psychological Association. (1994). Diagnostic and statistical manual of mental disorders $\left(4^{\text {th }}\right.$ Edition $)$. Washington, DC: Author.

Beck, A.T. (1976).Cognitive therapy and the emotional disorders. New York: International Universities Press.

Beck, J.S., \& Steer, R.A. (1990).Beck anxiety inventory manual. San Antonio, TX: Psychological Corporation.

Beck, J.S., Steer, R.A., Ball, R., \& Ranieri, W.F. (1996). Comparison of Beck depression inventories I and II in psychiatric outpatients. Journal of Personality Assessment, 67, 588-597.

Beck, J.S., Steer, R.A., \& Brown, G.K. (1996). Manual for the Beck depression inventory-II. San Antonio, TX: PsychologicalCorporation.

Bobes, J., Gonzalez, M.P., Bascaran, M.T., Arango, C., Saiz, P.A., \& Bousono, M. (2001). Quality of life and disability in patients with obsessive-compulsive disorder. European Psychiatry, 16, 239-245.

Brown, T.A., DiNardo, P., \& Barlow, D.H. (1994). Anxiety disorders interview schedule adult version (ADIS-IV): Client interview schedule. Oxford: Oxford University Press. 
Chambless, D.L., \& Gillis, M.M. (1993). Cognitive therapy of anxiety disorders. Journal of Consulting and Clinical Psychology, 61, 248-260.

Clark, D.A. (2004). Cognitive-behavioural therapy for OCD. New York: Guilford Press.

Cottraux, J., Bouvard, M.A., \& Milhery, M. (2005). Combining pharmacotherapy with cognitive-behavioural interventions for obsessive-compulsive disorder. Cognitive Behaviour Therapy, 34, 185-192.

Coughtrey, A.E., Shafran, R., Knibbs, D. \& Rachman, S.J. (submitted). Mental contamination in Obsessive Compulsive Disorder. Manuscript submitted for publication.

Eddy, K., Dutra, L., Bradley, R., \& Western, D. (2004). A multi-dimensional meta-analysis of psychotherapy and pharmacotherapy for OCD. Clinical Psychology Review, 24, 1011-1030.

Freeston, M.H., Ladouceur, R., Gagnon, F., Thibodeau, N., Rheaume, J., Letarte, H., \& Bujold, A. (1997). Cognitive-behavioural treatment of obsessive thoughts: A controlled study. Journal of Consulting and Clinical Psychology, 65, 405-413.

Fisher, P., \& Wells, A. (2005). How effective are cognitive and behavioural treatments for OCD? A clinical significance analysis. Behaviour Research and Therapy, 43, 15431558.

Foa, E.B., Huppert, J.D., Leiberg, S., Langner, R., Kichic, R., Hajcak, G., \& Salkovskis, P.M. (2002). The obsessive-compulsive inventory: Development and validation of a short version. Psychological Assessment, 14, 485-496. 
Foa, E.B., Liebowitz, M.R., Kozak, M.J., Davies, S., Campeas, R., Franklin, M.E., Huppert, J.D., Kjernisted, K., Rowan, V., Schmidt, A.B., Simpson, H.B., \& Tu, X. (2005). Randomised, placebo-controlled trial of exposure and ritual prevention, clomipramine, and their combination in the treatment of obsessive-compulsive disorder. American Journal of Psychiatry, 162, 151-161.

Goodman, W.K., Price, L.H., Rasmussen, S.A., Mazure, C., Fleischmann, R.L., Hill, C.L., Heninger, G.R., \& Charney, D.S. (1989). The Yale-Brown obsessive compulsive scale: Development, use and validity. Archives of General Psychiatry, 46, 1006-1011.

Jacobi, D., Herba, J., \& Rachman, S.J. (2005). Vancouver University Hospital: Anxiety Disorders Clinic, unpublished data.

Jacobson, N.S., \& Truax, P. (1991). Clinical significance: A statistical approach to defining meaningful change in psychotherapy research. Journal of Consulting and Clinical Psychology, 59, 12-19.

Jenike, M.A., Baer, L., \& Minichiello, W.E. (1998). Obsessive-compulsive disorders: Practical management. St. Louis: Mosby.

Kessler, R.C., Chiu, W.T., Demler, O., \& Walters, E.E. (2005).Prevalence, severity, and comorbidity of 12-month DSM-IV disorders in the national comorbidity survey replication. Archives of General Psychiatry, 62, 617-627.

Kim, S., Dysken, M., \& Kuskowski, M. (1990).The Yale-Brown obsessive compulsive scale: A reliability and validity study. Psychiatry Research, 41, 37-44.

National Institute for Health and Clinical Excellence (2005). Core interventions in the treatment of obsessive-compulsive disorder and body dysmorphic disorder. National Clinical Practice Guideline Number 31. 
Niederauer, K.G., Braga, D.T., de Souza, F.P., Meyer, E., \& Cordioli, A.V. (2007). Quality of life in patients with obsessive-compulsive disorder: A review. RevistaBrasileira de Psiquiatria, 29, 271-278.

Rachman, S.J. (2006). The fear of contamination: Assessment and treatment. Oxford: Oxford University Press.

Rachman, S.J. (2010). Betrayal: A psychological analysis. Behaviour, Research and Therapy, 48, 304-311.

Rachman, S.J., Shafran, R., Mitchell, D., Trant, J., \& Teachman, B. (1996). How to remain neutral: An experimental analysis of neutralisation. Behaviour Research and Therapy, 34, 889-898.

Radomsky, A. \& Elliott, C. M. (2009). Analyses of mental contamination: Part II, individual differences. Behaviour, Research and Therapy, 47, 1004-1011.

Riley, C., Lee, M., Cooper, Z., Fairburn, C.G., \& Shafran, R. (2007). A randomised controlled trial of cognitive behaviour therapy for clinical perfectionism: A preliminary study. Behaviour, Research and Therapy, 45, 2221-2231.

Salkovskis, P.M. (2007). Cognitive-behavioural treatment for obsessional ruminations and rituals: How do they compare? Paper presented at the World Congress of Behavioural and Cognitive Therapies, Barcelona, Spain.

Salkovskis, P.M., \& Kirk, J. (1997). Obsessive-compulsive disorder. In D.M. Clark \& C Fairburn (Eds.) The science and practice of cognitive behaviour therapy. Oxford: Oxford University Press. 
Shafran, R., Thordarson, D.S., \& Rachman, S.J. (1996). Thought-action fusion in obsessivecompulsive disorder.Journal of Anxiety Disorders, 10, 379-391.

Shetti, C.N., Reddy, Y.C.J., Kandavel, T., Kashyap, K., Singisetti, S., Hiremath, A.S., Siddequehusen, M.U.F., \& Raghunandanan, S. (2005). Clinical predictors of drug nonresponse in obsessive-compulsive disorder.Journal of Clinical Psychiatry, 66, 15171523.

Sookman, D., Abramowitz, J.S., Calamari, J.E., Wilhelm, S., \& McKay, D. (2005). Subtypes of obsessive-compulsive disorder: Implications for specialised cognitive behaviour therapy. Behaviour Therapy, 36, 393-400.

Sookman, D. \& Steketee, G. (2007). Directions in specialised cognitive behaviour therapy for resistant obsessive-compulsive disorder: Theory and practice of two approaches. Cognitive and Behavioural Practice, 14, 1-17.

Stanley, M.A., \& Turner, S.M. (1995). Current status of pharmacological and behavioural treatment of obsessive-compulsive disorder. Behaviour Therapy, 26, 163-186.

Volz, C., \&Heyman, I. (2007). Case series: Transformation obsession in young people with obsessive-compulsive disorder (OCD). Journal of the American Academy of Child and Adolescent Psychiatry, 46, 766-772.

Whittal, M.L., Robichaud, M., Thordarson, D.S., \& McLean, P.D. (2008). Group and individual treatment of obsessive-compulsive disorder using cognitive therapy and exposure plus response prevention: A two year follow-up of two randomised trials. Journal of Consulting and Clinical Psychology, 76, 1003-1014. 
Whittal, M.L., Woody, S.R., McLean, P.D., Rachman, S.J., \& Robichaud, M. (2010). Treatment of obsessions: A randomised controlled trial. Behaviour Research and Therapy.

Wild, J., \& Clark, D.M. (2011). Imagery rescripting of early traumatic memories in social phobia. Cognitive and Behavioural Practice, 18, 433-443.

Wilhelm, S. (2000). Cognitive therapy for obsessive-compulsive disorder. Journal of Cognitive Psychotherapy, 14, 245-259.

Wroe, A.L., \& Salkovskis, P.M. (2000). Causing harm and allowing harm: A study of beliefs in obsessional problems. Behaviour Research and Therapy, 38, 1141-1162. 
Table 1. Scores at Pre-Treatment, Post-Treatment and Follow-Up

\begin{tabular}{|c|c|c|c|c|c|c|c|c|c|c|c|c|c|}
\hline & & $\mathrm{P} 1$ & $\mathrm{P} 2$ & P3 & $\mathrm{P} 4$ & $\mathrm{P} 5$ & P6 & P7 & P8 & P9 & P10 & P11 & P12 \\
\hline \multicolumn{2}{|c|}{ Participant Age } & 34 & 19 & 29 & 44 & 22 & 22 & 22 & 43 & 25 & 31 & 21 & 34 \\
\hline \multicolumn{2}{|c|}{ Participant Gender } & $\mathrm{F}$ & M & M & M & $\mathrm{F}$ & M & $\mathrm{F}$ & $\mathrm{F}$ & $\mathrm{F}$ & $\mathrm{F}$ & M & $\mathrm{F}$ \\
\hline Y-BOCS & Pre- & 29 & 29 & 30 & 34 & 27 & 33 & 29 & 32 & 21 & 28 & 26 & 29 \\
\hline \multirow[t]{4}{*}{ Total } & treatment & & & & & & & & & & & & \\
\hline & Post- & 6 & 32 & 1 & 35 & 22 & 2 & 1 & 28 & 3 & 24 & 3 & 2 \\
\hline & 3-month & 3 & - & $\mathbf{0}$ & - & - & 2 & 1 & 27 & 1 & - & 2 & 1 \\
\hline & 6-month & 4 & - & 4 & - & - & $\mathbf{0}$ & 1 & - & 2 & - & 1 & $\mathbf{1}$ \\
\hline \multirow[t]{6}{*}{ VOCI-MC } & Pre- & 68 & 41 & 45 & 94 & 52 & 40 & 52 & 74 & 46 & 55 & 59 & 59 \\
\hline & treatment & & & & & & & & & & & & \\
\hline & Post- & 13 & 15 & 5 & 83 & 49 & 2 & 8 & 76 & 3 & 15 & 4 & 6 \\
\hline & treatment & & & & & & & & & & & & \\
\hline & 3-month & 7 & - & 3 & - & - & $\mathbf{0}$ & 9 & 75 & 4 & - & 1 & 5 \\
\hline & 6-month & 8 & - & 4 & - & - & 4 & 8 & - & 2 & - & 11 & 8 \\
\hline
\end{tabular}

OCI-R

Pre-

$\begin{array}{lllll}45 & 34 & 55 & 56 & 27\end{array}$

$31 \quad 36 \quad 64$

$\begin{array}{llll}46 & 28 & 37 & 42\end{array}$


treatment

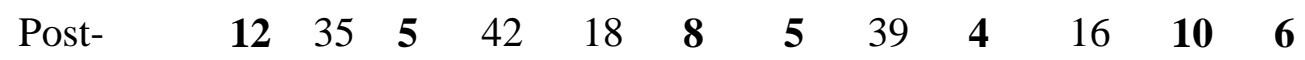

treatment

$\begin{array}{llllllllllllll}3-\text { month } & \mathbf{1 0} & - & \mathbf{6} & - & - & \mathbf{1 1} & \mathbf{7} & 42 & \mathbf{6} & - & \mathbf{3} & \mathbf{5}\end{array}$

follow-up

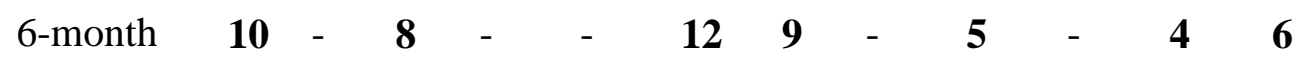

follow-up

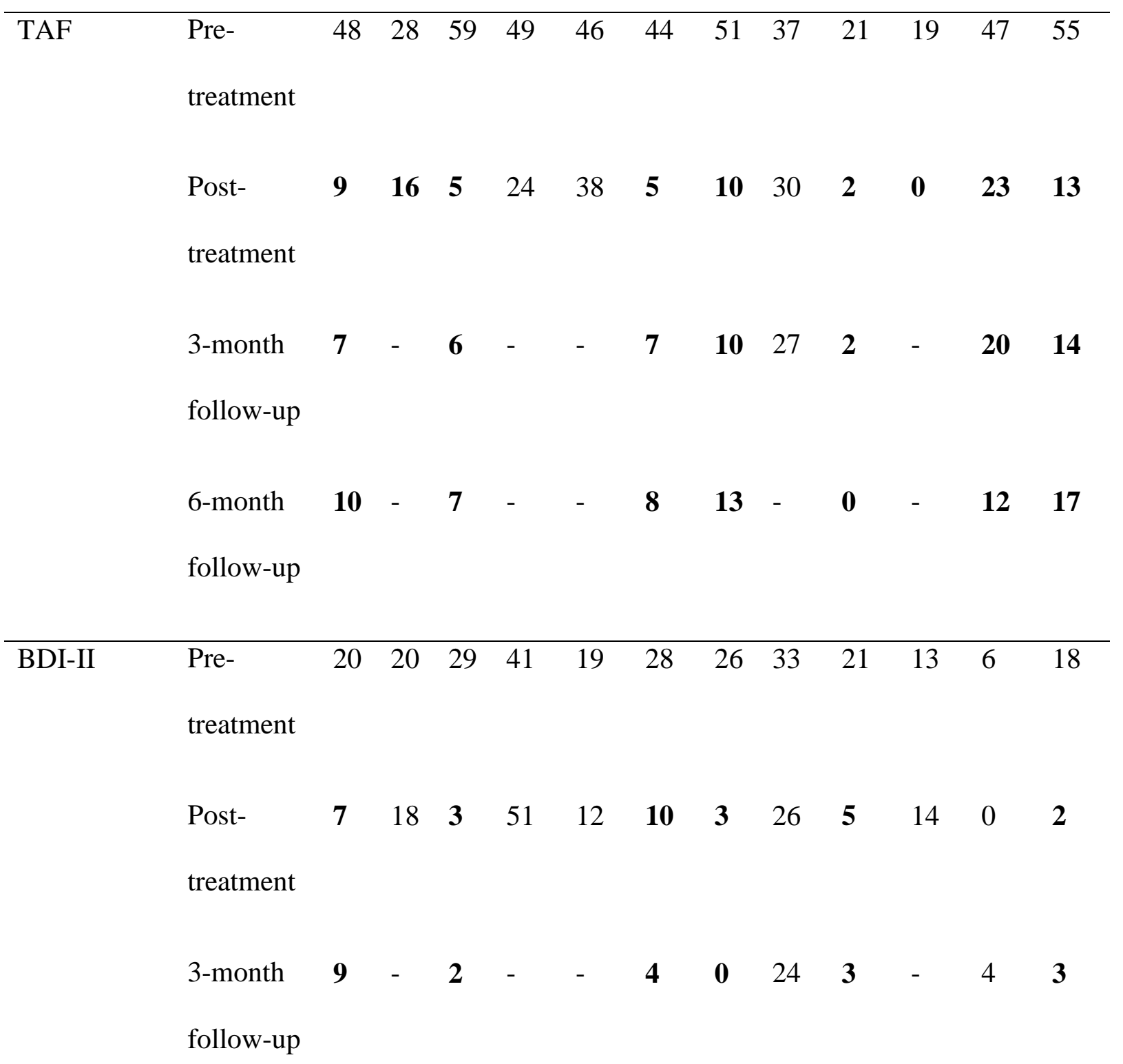




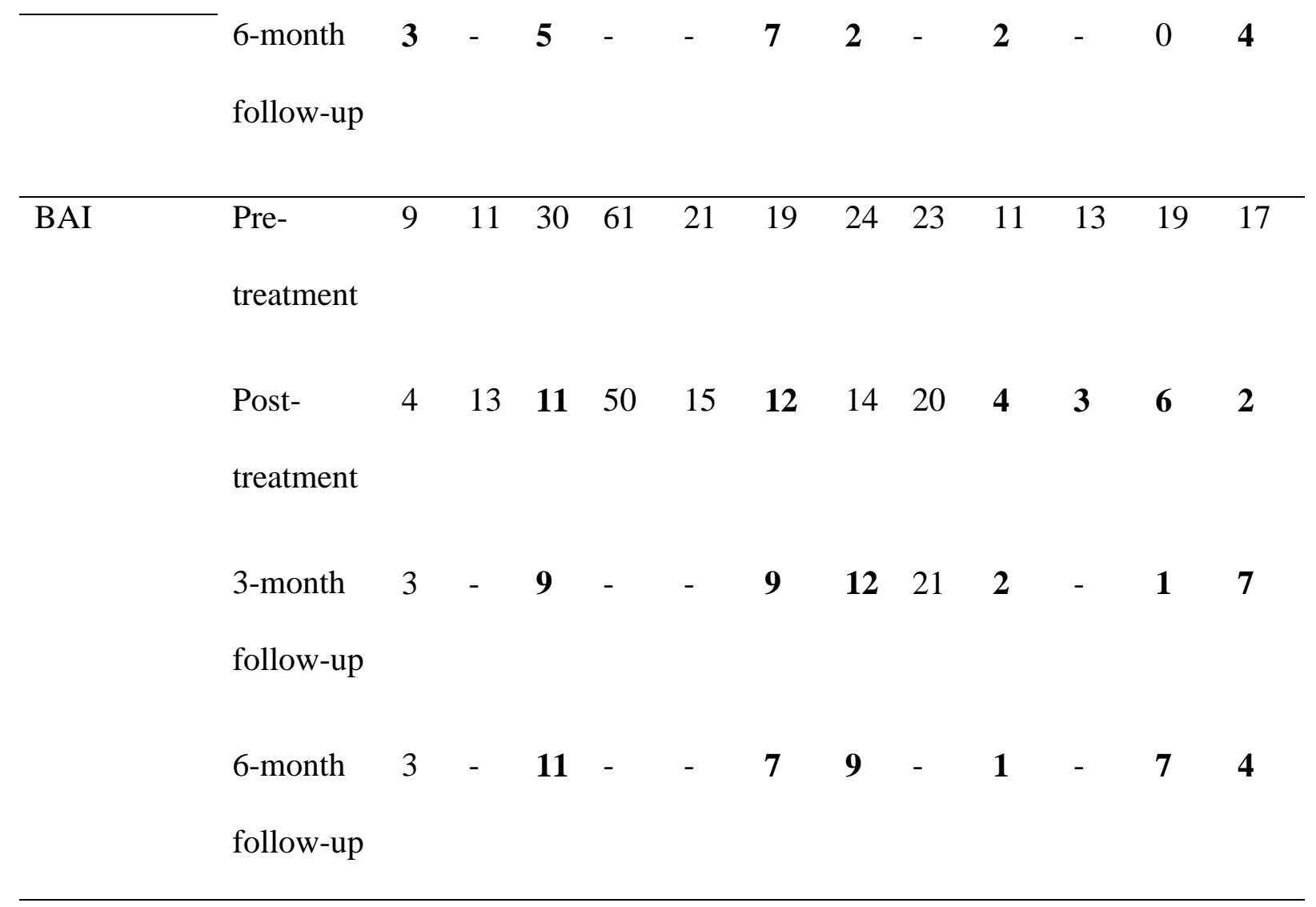

Note. Clinically significant change indicated by scores in bold. 
Table 2. Mean Scores Pre-Treatment, Post-Treatment and at 3 and 6 Month Follow-Up.

\begin{tabular}{|c|c|c|c|c|c|c|c|c|}
\hline & \multicolumn{2}{|c|}{ Pre-treatment } & \multicolumn{2}{|c|}{ Post-treatment } & \multicolumn{2}{|c|}{$3 \mathrm{~m}$ follow-up } & \multicolumn{2}{|c|}{$6 \mathrm{~m}$ follow-up } \\
\hline & $M$ & $S D$ & $M$ & $S D$ & $M$ & $S D$ & $M$ & $S D$ \\
\hline Y-BOCS & 29.00 & 3.38 & 13.42 & 13.78 & 12.75 & 14.15 & 12.92 & 14.04 \\
\hline OCI-R & 42.67 & 10.98 & 18.25 & 15.22 & 18.33 & 15.73 & 18.83 & 15.34 \\
\hline VOCI-MC & 57.92 & 15.49 & 24.00 & 29.98 & 22.92 & 30.46 & 24.25 & 29.57 \\
\hline TAF & 40.58 & 13.45 & 13.75 & 10.36 & 13.42 & 9.62 & 13.50 & 9.41 \\
\hline BDI-II & 22.67 & 9.37 & 13.08 & 14.34 & 12.50 & 14.40 & 12.33 & 14.46 \\
\hline BAI & 21.00 & 14.02 & 12.83 & 13.00 & 12.08 & 13.35 & 12.00 & 13.29 \\
\hline
\end{tabular}

